\section{ORIGINAL} RESEARCH

A. Chutinet

A. Biffi

A. Kanakis

K.M. Fitzpatrick

K.L. Furie

N.S. Rost

\title{
Severity of Leukoaraiosis in Large Vessel Atherosclerotic Disease
}

BACKGROUND AND PURPOSE: The severity of white matter hyperintensity, or leukoaraiosis, is a marker of cerebrovascular disease. In stroke, WMH burden is strongly linked to lacunar infarction; however, impaired cerebral perfusion due to extracranial or intracranial atherosclerosis may also contribute to WMH burden. We sought to determine whether WMH burden is associated with extracranial or intracranial stenosis in patients with AIS.

MATERIALS AND METHODS: Patients with AIS with admission head/neck CTA and brain MR imaging were included in this analysis. "Extracranial stenosis" was defined as $>50 \%$ stenosis in the extracranial ICA, and "intracranial," as $>50 \%$ stenosis in either the middle, anterior, or posterior cerebral arteries on CTA, on either side. WMHV was determined by using a validated semiautomated protocol. Multiple regression was used to assess the relationship between WMHV and extracranial/intracranial atherosclerosis.

RESULTS: Of 201 subjects, 51 (25.4\%) had extracranial and 63 (31.5\%) had intracranial stenosis. Mean age was $62 \pm 15$ years; $36 \%$ were women. Mean WMHV was $12.87 \mathrm{~cm}^{3}$ in the extracranial and 8.59 $\mathrm{cm}^{3}$ in the intracranial stenosis groups. In univariate analysis, age $(P<.0001)$, SBP and DBP $(P=.004)$, and HTN $(P=.0003)$ were associated with WMHV. Extracranial stenosis was associated with greater WMHV after adjustment for intracranial stenosis $(P=.04)$. In multivariate analysis including extracranial stenosis, only age $(P<.0001)$ and HTN $(P=.03)$ demonstrated independent effects on WMHV.

CONCLUSIONS: In our cohort of patients with AIS, age and HTN were the strongest determinants of the WMHV severity. Future studies are warranted to unravel further association between WMHV and cerebral vessel atherosclerosis.

ABBREVIATIONS: $\mathrm{AF}=$ atrial fibrillation; $\mathrm{AIS}=$ acute ischemic stroke; $\mathrm{CAD}=$ coronary artery disease; $\mathrm{DBP}=$ diastolic blood pressure; $\mathrm{DM}=$ diabetes mellitus; HTN = hypertension; $\mathrm{IQR}=$ interquartile range; InWMHV = natural logarithm-transformed white matter hyperintensity volume; $\mathrm{SBP}=$ systolic blood pressure; TOAST = Trial of Org 10172 in Acute Stroke Treatment; WMH = white matter hyperintensity; WMHV = white matter hyperintensity volume

eukoaraiosis, also known as WMH, is commonly found in - patients with stroke or TIA. ${ }^{1}$ In patients with AIS, the severity of leukoaraiosis is associated with infarct growth, poor functional outcomes, and increased risk of recurrent stroke. ${ }^{1-4}$ Furthermore, leukoaraiosis has been strongly linked to smallvessel disease or lacunar infarction. ${ }^{5-7}$

However, impaired cerebral perfusion due to large-vessel atherosclerosis may affect the severity of leukoaraiosis. To date, association of leukoaraiosis and carotid stenosis in population-based studies remains controversial. ${ }^{8-11}$ Among patients with ischemic stroke, an association between WMH severity and large-artery stroke subtype was reported in a single study of Korean patients, ${ }^{12}$ whereas other studies demon-

Received September 22, 2011; accepted after revision November 10.

From the J. Philip Kistler Stroke Research Center (A.C., A.B., A.K., K.M.F., K.L.F., N.S.R.), Department of Neurology, Massachusetts General Hospital, Boston, Massachusetts; and Division of Neurology (A.C.), Department of Medicine, Faculty of Medicine, Chulalongkorn University, Bangkok, Thailand.

This work was supported by the National Institutes of Health-National Institute of Neurological Disorders and Stroke (K23NS064052, N.S.R.; P50NS051343, K.L.F.), the American Heart Association/Bugher Foundation Centers for Stroke Prevention Research (0775010N), and the Deane Institute for Integrative Study of Atrial Fibrillation and Stroke.

Please address correspondence to Natalia S. Rost, MD, MPH, J. Philip Kistler Stroke Research Center, Massachusetts General Hospital, 175 Cambridge St, Suite 300, Boston MA 02114; e-mail: nrost@partners.org

Indicates open access to non-subscribers at www.ajnr.org

http://dx.doi.org/10.3174/ajnr.A3015 strated greater prevalence of leukoaraiosis in intracranial rather than extracranial atherosclerotic disease of the cerebral vasculature. $^{12,13}$

We sought to determine whether the burden of WMH measured as MR imaging-detectable WMHV is associated with the radiographic evidence of extracranial or intracranial stenosis in patients with AIS.

\section{Materials and Methods}

\section{Study Population}

We selected subjects from consecutive patients with AIS older than 18 years of age with CTA of head and neck and a brain MR imaging completed on admission to our hospital between January 1, 2008, and January 1, 2009. A total of 201 subjects met the inclusion criteria. "Ischemic stroke" was defined as an acute vascular syndrome with evidence of cerebral infarction on DWI completed within 48 hours from symptom onset.

All patients were assessed prospectively on admission and at 3month follow-up. On admission, the NIHSS score was assessed as part of the AIS evaluation in the emergency department. Vascular risk factors, including HTN, DM, hyperlipidemia, CAD, and AF, were collected by patient or proxy interview or medical record review. Variables were defined as in prior publications. ${ }^{14}$ The first available SBP, DBP, and creatinine levels measured on admission were recorded. Stroke subtypes were classified by using the TOAST criteria, which included large-artery atherosclerosis (embolus/thrombosis), 


\begin{tabular}{|c|c|}
\hline \multicolumn{2}{|l|}{ Baseline Characteristics } \\
\hline Age (yr) (mean \pm SD) & $62.45 \pm 15.02$ \\
\hline Female (No.) (\%) & $72(35.82)$ \\
\hline HTN (No.) (\%) & $119(59.20)$ \\
\hline DM (№.) (\%) & $36(17.91)$ \\
\hline Smoking (№.) (\%) & $119(60.1)$ \\
\hline Hyperlipidemia (№.) (\%) & $84(41.79)$ \\
\hline CAD (No.) (\%) & $34(16.92)$ \\
\hline $\mathrm{AF}(\mathrm{No}).(\%)$ & $18(8.96)$ \\
\hline Extracranial carotid stenosis (№.) (\%) & $51(25.37)$ \\
\hline Intracranial arterial stenosis (№.) (\%) & $63(31.50)$ \\
\hline WMHV $\left(\mathrm{cm}^{3}\right)$ (median) (IOR) & $5.0(2.5-10.68)$ \\
\hline InWMHV (mean \pm SD) & $1.61 \pm 1.19$ \\
\hline WMHV (extracranial stenosis) $\left(\mathrm{cm}^{3}\right)($ mean \pm SD) & $12.87 \pm 2.4$ \\
\hline WMHV (intracranial stenosis) $\left(\mathrm{cm}^{3}\right)($ mean $\pm \mathrm{SD})$ & $8.59 \pm 1.6$ \\
\hline $\mathrm{SBP}(\mathrm{mm} \mathrm{Hg})($ mean $\pm \mathrm{SD})$ & $151.54 \pm 29.23$ \\
\hline $\mathrm{DBP}(\mathrm{mm} \mathrm{Hg})($ mean $\pm \mathrm{SD})$ & $81.43 \pm 14.70$ \\
\hline Creatinine level (mg/dL) (median) (IOR) & $1.0(0.84-1.17)$ \\
\hline NIHSS score, median (IQR) & $2.0(1-14)$ \\
\hline \multicolumn{2}{|l|}{ TOAST classification } \\
\hline Large-artery atherosclerosis (№.) (\%) & $50(25.00)$ \\
\hline Cardioembolism (№.) (\%) & $80(40.00)$ \\
\hline Small-vessel occlusion (No.) (\%) & $27(13.50)$ \\
\hline Undetermined etiology (№.) (\%) & $36(18.00)$ \\
\hline Other determined etiology (No.) (\%) & $8(4.00)$ \\
\hline
\end{tabular}

cardioembolism (high-risk/medium-risk), small-vessel occlusion (LACUNE), stroke of other determined etiology, and stroke of undetermined etiology (Table 1). ${ }^{15}$ All patients or their health care proxy gave informed consent to take part in the ongoing prospective hospital-based cohort studies that were approved by the local institutional review board.

\section{Neuroimaging Analysis}

CTA of the head and neck was performed by using a helical scanner (HiSpeed Advantage; GE Healthcare, Milwaukee, Wisconsin) as part of the emergency AIS evaluation. "Extracranial stenosis" was defined as evidence of $>50 \%$ stenosis in the extracranial ICA based on NASCET criteria, ${ }^{16}$ and "intracranial," as $>50 \%$ stenosis in either the middle (M1), anterior (A1), or posterior cerebral arteries (P1) on CTA, on either side (Fig 1). CTA measurements were performed by a reader (A.C.) blinded to clinical data and WMHV measurements.

MR imaging was performed by using $1.5 \mathrm{~T}$ scanners (Signa, GE Healthcare). We used MRIcro software (University of Nottingham School of Psychology, Nottingham, UK; www.mricro.com) to convert scans from DICOM into Analyze format and for computerassisted determination of WMHV. ${ }^{17-19}$ Volumetric analysis of WMHV was performed on the T2 FLAIR axial sequences using a previously described semiautomated method (Fig 2). ${ }^{18-20}$ To avoid confounding by the presence of focal white matter changes due to index stroke and to aid discrimination of infarcts from $\mathrm{WMH}$, we simultaneously viewed DWI, ADC, and T2 FLAIR sequences. Total WMHV was measured from the hemisphere unaffected by AIS based on the previously validated protocol. ${ }^{18}$ All WMHV measurements were performed by readers blinded to clinical data and the results of large-vessel stenosis assessment.


Fig 1. CTA definition of extracranial and intracranial largevessel stenosis. Compared with the nonobstructive ICA disease $(A)$, extracranial carotid artery stenosis was defined as evidence of $>50 \%$ stenosis based on the NASCET criteria (B). Similarly, compared with the large intracranial vessel disease $(<50 \%, M 1, C)$, intracranial stenosis was defined as $>50 \%$ stenosis in either the middle (M1) (D), anterior (A1), or posterior cerebral arteries (P1) on CTA, on either side.
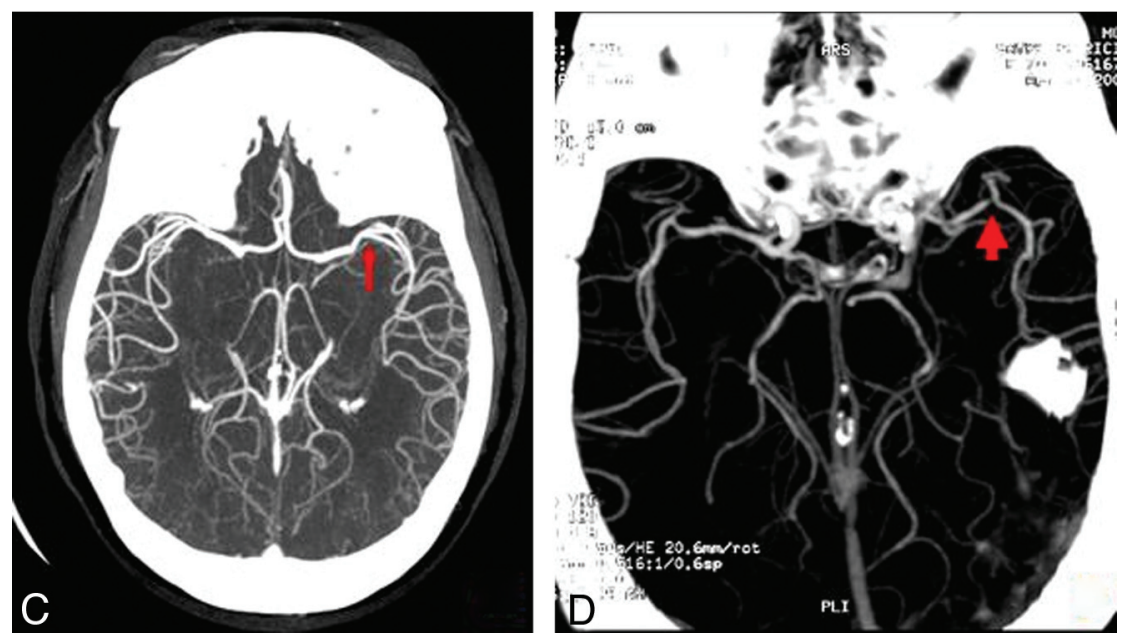

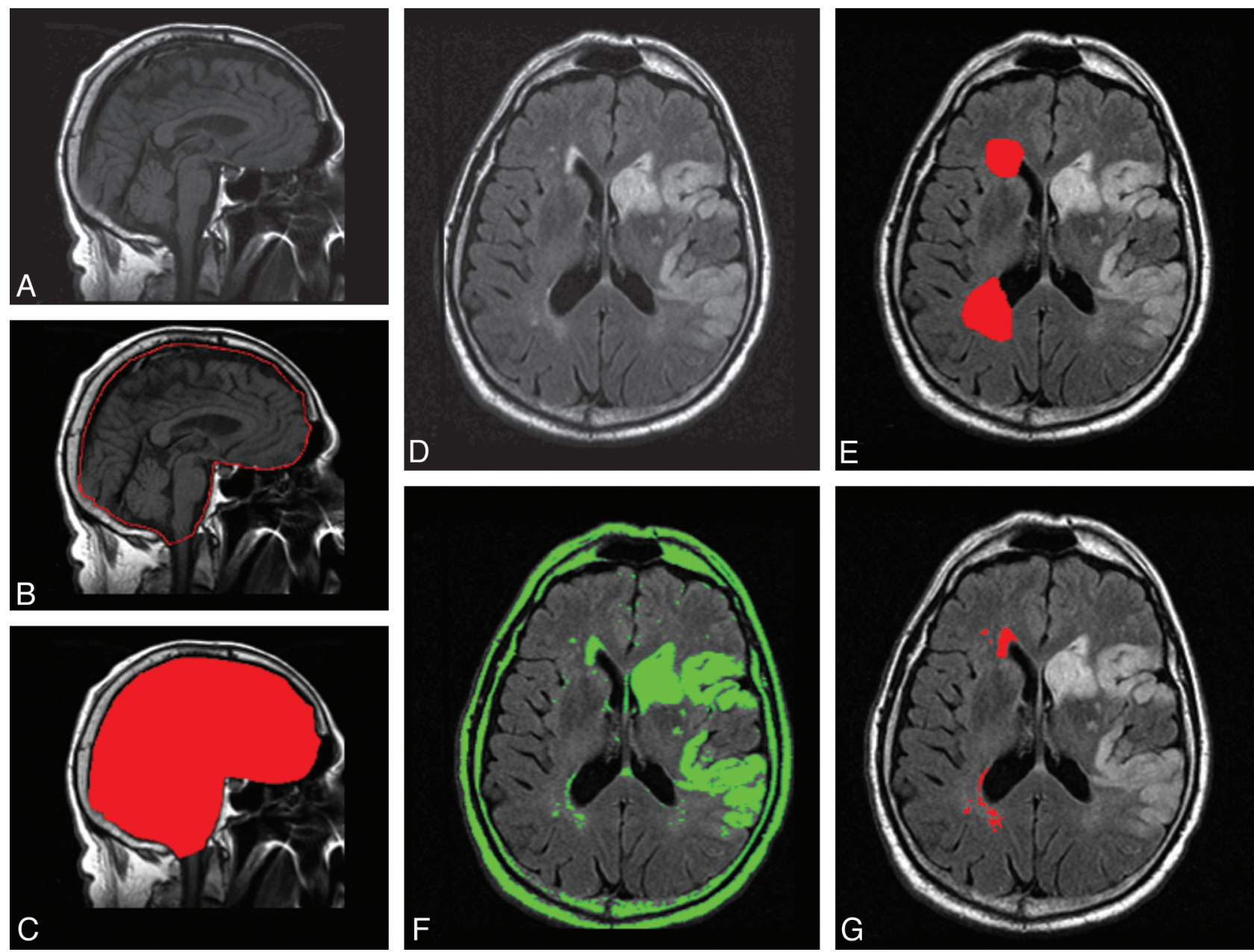

Fig 2. Volumetric analysis of WMH in patients with AIS. Left: for each subject, the T1 sagittal sequence of admission brain MR imaging $(A)$ is used to outline the 2 adjacent median sections $(B)$. The combined volume of these sections is used to calculate the intracranial area $(C)$, a validated marker of intracranial volume. Right: T2 FLAIR axial sequences are examined for the presence of WMH $(D)$. Regions of interest representing leukoaraiosis are outlined in the hemisphere contralateral to the acute infarct $(E)$, and a semiautomated intensity filter is applied to all consecutive supratentorial axial FLAIR sections (F). The intersection between the initial region of interest and the intensity filter is examined by an operator trained to edit the final region of interest $(G)$ and to finalize the WMH volume calculations adjusted for head size by using the intracranial area.

\section{Statistical Analysis}

All continuous numeric variables are expressed as mean \pm SD, with the exception of WMHV, creatinine level, and NIHSS scores, which are expressed as medians (IQR). WMHV was normalized for linear regression analysis by using $\ln W \mathrm{MHV}$. Univariate linear regression analysis was used to analyze the relationship between dichotomous and continuous variables and lnWMHV. Three multivariate linear regression models were built to test for independent predictors of $\ln \mathrm{W}$ MHV. Model 1 included all univariate predictors with $P<.2$. Model 2 included the additionally forced in intracranial arterial stenosis, whereas model 3 was an exploratory model of the effect of a subtype of large-artery stenosis on WMH burden. $P$ value $<.05$ was considered significant for all analyses. All statistical analyses were performed by using SAS software (SAS 9.2; SAS Institute, Cary, North Carolina).

\section{Results}

A total of 201 consecutive patients with AIS with admission head and neck CTA and brain MR imaging suitable for WMH volumetric analysis were included in the study. Baseline characteristics are shown in Table 1 . Mean age was $62 \pm 15$ years; $36 \%$ were women. Smoking $(60.1 \%)$ and HTN (59\%) were the leading vascular risk factors in this cohort, followed by hyperlipidemia (42\%). Among these patients, 80 (40\%) had cardioembolic, 50 (25\%) had large-artery, 36 (18\%) had undetermined, $27(13.5 \%)$ had small-vessel, and $8(4 \%)$ had other TOAST stroke subtypes.

Median WMHV was $5 \mathrm{~cm}^{3}$ (IQR, 2.5-10.7). Based on predefined CTA criteria, 51/201 patients (25\%) had extracranial, whereas 63/201 (32\%) had intracranial stenosis. Mean WMHV was greater in patients with extracranial $\left(12.87 \mathrm{~cm}^{3}\right)$ compared with intracranial stenosis $\left(8.59 \mathrm{~cm}^{3}\right)$.

In univariate analysis, age, SBP, DBP, and history of HTN were significantly associated with $\ln \mathrm{WMHV}(P<.0001$, $.0003, .0041$, and .0003 , respectively), whereas extracranial stenosis demonstrated a trend for association with lnWMHV $(P=.05)$ (Table 2).

In multivariate analysis (Table 3), age and SBP (both $P<$ $.0001)$ were independently associated with $\operatorname{lnWMHV}$ in model 1. Including intracranial stenosis into multivariable regression did not alter the results (model 2). Adjusting for the independent effect of extracranial and intracranial stenosis on WMH burden in model 3 demonstrated that extracranial but not intracranial stenosis was independently associated with $\operatorname{lnWMHV}(P=.04)$. 


\begin{tabular}{|c|c|c|c|}
\hline Predictors & Estimate & T Value & $P$ Value \\
\hline Age (yr) & 0.04 & 7.70 & $<.0001^{a}$ \\
\hline Sex & 0.27 & 1.54 & .13 \\
\hline SBP & 0.01 & 3.69 & $.0003^{\mathrm{a}}$ \\
\hline DBP & 0.02 & 2.91 & $.0041^{\mathrm{a}}$ \\
\hline Creatinine level & 0.32 & 1.23 & .22 \\
\hline \multicolumn{4}{|l|}{ Medical history } \\
\hline HTN & 0.62 & 3.72 & $.000^{\mathrm{a}}$ \\
\hline $\mathrm{DM}$ & 0.17 & 0.76 & 0.45 \\
\hline Smoking & 0.08 & 0.51 & 0.61 \\
\hline Hyperlipidemia & -0.02 & -0.12 & 0.90 \\
\hline CAD & 0.03 & 0.13 & 0.90 \\
\hline AF & 0.25 & 0.84 & 0.40 \\
\hline \multicolumn{4}{|l|}{ Site of vascular stenosis } \\
\hline Extracranial carotid stenosis & 0.37 & 1.93 & 0.05 \\
\hline Intracranial arterial stenosis & 0.12 & 0.66 & 0.51 \\
\hline
\end{tabular}

\begin{tabular}{|c|c|c|c|}
\hline Predictors & Estimate & T Value & $P$ Value \\
\hline Model $1^{a}$ & & $F=13.42$ & $<.0001$ \\
\hline Age (yr) & 0.03 & 6.34 & $<.0001^{d}$ \\
\hline Sex & 0.07 & 0.45 & .65 \\
\hline SBP & 0.01 & 2.24 & $.03^{\mathrm{d}}$ \\
\hline HTN & 0.12 & 0.68 & .50 \\
\hline Extracranial carotid stenosis & 0.03 & 0.16 & .87 \\
\hline Model $2^{b}$ & & $F=11.45$ & $<.0001$ \\
\hline Age (yr) & 0.03 & 6.19 & $<.0001^{\mathrm{d}}$ \\
\hline Sex & 0.11 & 0.66 & .51 \\
\hline SBP & 0.01 & 2.43 & $.02^{\mathrm{d}}$ \\
\hline HTN & 0.14 & 0.79 & .43 \\
\hline Extracranial carotid stenosis & 0.11 & 0.60 & .55 \\
\hline Intracranial arterial stenosis & -0.12 & -0.69 & .49 \\
\hline Model $3^{c}$ & & $F=2.35$ & .10 \\
\hline Intracranial arterial stenosis & -0.01 & -0.05 & .96 \\
\hline Extracranial carotid stenosis & 0.42 & 2.06 & $.04^{\mathrm{d}}$ \\
\hline
\end{tabular}

a Model includes all univariate predictors with $P<0.2$.

${ }^{b}$ Model includes all univariate predictors with $P<0.2$ and intracranial arterial stenosis.

${ }^{c}$ Exploratory model of the effect of subtype of large-artery stenosis on white matter

hyperintensity burden.

Statistically significant.

\section{Discussion}

In our study population, age and HTN were the independent predictors of WMH severity in patients with AIS. Increasing age is the strongest predictor of leukoaraiosis burden in general populations ${ }^{11,21-25}$ and in patients with ischemic stroke. ${ }^{12,13,26}$ Furthermore, there is pathologic evidence linking age and WMH lesions. ${ }^{27}$ Proposed mechanisms of association between leukoaraiosis and advancing age include the following: 1) alteration of blood supply to the white matter due to arteriosclerosis, tortuosity, and elongation of the vessels that result from the aging process ${ }^{23,28-33}$; and 2) age-related changes to the periventricular white matter regions that are in the arterial borderzone and easily susceptible to systemic or focal decreases in cerebral blood flow. ${ }^{34}$

Besides age, HTN is the most common risk factor for WMH. The association of elevated blood pressure and severity of WMH was demonstrated in previous population-based studies ${ }^{21-25}$ and in patients with ischemic stroke, including our results. ${ }^{12-13,35,36}$ Despite differences in populations evaluated, our results are consistent with the Atherosclerosis Risk in
Communities study, which demonstrated that the level of SBP is related to $\mathrm{WMH}$ severity. ${ }^{35,37}$ Moreover, longitudinal investigations found that well-controlled blood pressure in patients with HTN can reduce the risk of severe WMH lesions. ${ }^{38,39}$ Chronically elevated blood pressure changes small penetrating arteries and arterioles of the white matter by replacing the smooth muscle cells with fibrohyaline material, which, in turn, causes arteriosclerosis and, presumably, leukoaraiosis. ${ }^{33,39}$

Although previous studies showed that leukoaraiosis is strongly related to small-vessel disease, ${ }^{5-7}$ conflicting findings regarding the association between WMH and AIS subtypes have been reported. A study in Korean patients with ischemic stroke found that leukoaraiosis was predominately associated with large-artery stroke subtype. ${ }^{12}$ In that study, WMH burden was assessed by using an ordinal visual scale, and prevalence of severe $\mathrm{WMH}$ was 55\% in large-vessel, $30 \%$ in smallvessel, and $14 \%$ in cardioembolic stroke subtype groups. On the other hand, a study in Chinese patients with AIS did not show a relationship between large-artery stenosis and WMH lesion burden. ${ }^{36}$ Most interesting, none of the previously published studies implicated diseased posterior cerebral vasculature in WMH severity or its burden accumulation.

Our study aimed to determine whether pre-existing largevessel stenosis predicted WMH severity independent of AIS subtype. We demonstrated that extracranial carotid stenosis was associated with higher WMHV compared with intracranial arterial stenosis. Our results differ from those in 2 prior studies in Korean patients with AIS, which reported WMH lesions to be more prevalent in intracranial arterial stenosis versus extracranial carotid stenosis. ${ }^{12,13}$ This difference may partially be explained by the overall prevalence of extracranial carotid stenosis in white populations, whereas intracranial stenosis is known to be common in Asians. ${ }^{40-44}$ Some other explanations of the divergence in our results include using the different method of WMHV measurement (volumetric assessment in our study versus an ordinal visual rating scale) as well as the definition of the large-vessel stenosis (CTA evaluation in our study versus MR angiography). ${ }^{12,13}$ Furthermore, the mechanism of extracranial carotid stenosis related to the severity of WMH might be through its link to microembolism of unstable plaques and other hemodynamic factors associated with extracranial carotid stenosis. A study in patients with symptomatic carotid stenosis showed an association between unstable (type IV: ulcerated surface, rupture, and hemorrhage) carotid plaques and WMH lesion numbers. ${ }^{45,46}$

The strength of our study is in using a validated highly accurate semiautomated protocol of WMHV assessment that allows detailed quantification of the WMH burden and stringent quality control. Furthermore, we used CTA to determine the degree of large-vessel stenosis, which is also known to have higher accuracy, compared with other noninvasive methods. ${ }^{16,47}$ The limitations of our methods include relatively constrained technical ability to separate WMH resulting from chronic lacunar strokes versus that of "true" leukoaraiosis by using conventional MR imaging sequences. However, the overall accuracy of the WMH measurement by using our operator-mediated quantitative approach is reassuring with regard to its specificity. Other limitations include the inherent drawbacks associated with a retrospective study design and a 
relatively small study size, which likely limits our study power. However, despite its modest size, our study may provide important clues to the direction of future research in this important area.

\section{Conclusions}

Atherosclerosis of the cerebral vasculature may play an important role in the severity of WMH among patients with ischemic stroke and large-artery disease. In our cohort of patients with AIS, age and HTN were the strongest determinants of the WMHV severity; however, future studies are warranted to investigate whether this link has an independent and significant role in the biology of the WMH disease in this patient population and across various ethnicities.

Disclosures: Alessandro Biffi—UNRELATED: Grants/Grants Pending: American Heart Association. Karen Furie-UNRELATED: Consultancy. Quintiles.

\section{References}

1. Ay H, Arsava EM, Rosand J, et al. Severity of leukoaraiosis and susceptibility to infarct growth in acute stroke. Stroke 2008;39:1409-13

2. Kissela B, Lindsell CJ, Kleindorfer D, et al. Clinical prediction of functional outcome after ischemic stroke: the surprising importance of periventricular white matter disease and race. Stroke 2009;40:530-36

3. Arsava EM, Rahman R, Rosand J, et al. Severity of leukoaraiosis correlates with clinical outcome after ischemic stroke. Neurology 2009;72:1403-10

4. van Swieten JC, Kappelle LJ, Algra A, et al. Hypodensity of the cerebral white matter in patients with transient ischemic attack or minor stroke: influence on the rate of subsequent stroke-Dutch TIA Trial Study Group. Ann Neurol 1992;32:177-83

5. Leys D, Englund E, Del Ser T, et al. White matter changes in stroke patients: relationship with stroke subtype and outcome. Eur Neurol 1999;42:67-75

6. Hijdra A, Verbeeten B Jr, Verhulst JA. Relation of leukoaraiosis to lesion type in stroke patients. Stroke 1990;21:890-94

7. Rost NS, Rahman RM, Biffi A, et al. White matter hyperintensity volume is increased in small vessel stroke subtypes. Neurology 2010;75:1670-77

8. Bots ML, van Swieten JC, Breteler MM, et al. Cerebral white matter lesions and atherosclerosis in the Rotterdam Study. Lancet 1993;341:1232-37

9. Streifler JY, Eliasziw M, Benavente OR, et al. Lack of relationship between leukoaraiosis and carotid artery disease: the North American Symptomatic Carotid Endarterectomy Trial. Arch Neurol 1995;52:21-24

10. de Leeuw FE, de Groot JC, Bots ML, et al. Carotid atherosclerosis and cerebral white matter lesions in a population based magnetic resonance imaging study. J Neurol 2000;247:2919-26

11. Pico F, Dufouil C, Levy C, et al. Longitudinal study of carotid atherosclerosis and white matter hyperintensities: the EVA-MRI cohort. Cerebrovasc Dis 2002;14:109-15

12. Lee SJ, Kim JS, Lee KS, et al. The leukoaraiosis is more prevalent in the large artery atherosclerosis stroke subtype among Korean patients with ischemic stroke. BMC Neurol 2008;8:31

13. Lee SJ, Kim JS, Chung SW, et al. White matter hyperintensities (WMH) are associated with intracranial atherosclerosis rather than extracranial atherosclerosis. Arch Gerontol Geriatr 2011;53:e129-32. Epub 2010 Aug 30

14. Jimenez-Conde J, Biffi A, Rahman R, et al. Hyperlipidemia and reduced white matter hyperintensity volume in patients with ischemic stroke. Stroke 2010;41:437-42

15. Adams HP Jr, Bendixen BH, Kappelle LJ, et al. Classification of subtype of acute ischemic stroke: definitions for use in a multicenter clinical trial-TOAST. Trial of Org 10172 in Acute Stroke Treatment. Stroke 1993;24:35-41

16. Brott TG, Halperin JL, Abbara S, et al. 2011 ASA/ACCF/AHA/AANN/AANS/ ACR/ASNR/CNS/SAIP/ SCAI/SIR/SNIS/SVM/SVS guideline on the management of patients with extracranial carotid and vertebral artery disease: executive summary - a report of the American College of Cardiology Foundation/ American Heart Association Task Force on Practice Guidelines, and the American Stroke Association, American Association of Neuroscience Nurses, American Association of Neurological Surgeons, American College of Radiology, American Society of Neuroradiology, Congress of Neurological Surgeons, Society of Atherosclerosis Imaging and Prevention, Society for Cardiovascular Angiography and Interventions, Society of Interventional Radiology, Society of NeuroInterventional Surgery, Society for Vascular Medicine, and Society for Vascular Surgery. Vasc Med 2011;16:35-77

17. Smith EE, Gurol ME, Eng JA, et al. White matter lesions, cognition, and recur- rent hemorrhage in lobar intracerebral hemorrhage. Neurology 2004;63:1606-12

18. Gurol ME, Irizarry MC, Smith EE, et al. Plasma beta-amyloid and white matter lesions in $\mathrm{AD}, \mathrm{MCI}$, and cerebral amyloid angiopathy. Neurology 2006;66:23-29

19. Chen YW, Gurol ME, Rosand J, et al. Progression of white matter lesions and hemorrhages in cerebral amyloid angiopathy. Neurology 2006;67:83-87

20. Nandigam RN, Chen YW, Gurol ME, et al. Validation of intracranial area as a surrogate measure of intracranial volume when using clinical MRI. J Neuroimaging 2007;17:74-77

21. Breteler MM, van Swieten JC, Bots ML, et al. Cerebral white matter lesions, vascular risk factors, and cognitive function in a population-based study: the Rotterdam Study. Neurology 1994;44:1246-52

22. Manolio TA, Kronmal RA, Burke GL, et al. Magnetic resonance abnormalities and cardiovascular disease in older adults: the Cardiovascular Health Study. Stroke 1994;25:318-27

23. Longstreth WT Jr, Manolio TA, Arnold A, et al. Clinical correlates of white matter findings on cranial magnetic resonance imaging of 3301 elderly people: the Cardiovascular Health Study. Stroke 1996;27:1274-82

24. Wong TY, Klein R, Sharrett AR, et al. Cerebral white matter lesions, retinopathy, and incident clinical stroke. JAMA 2002;288:67-74

25. Jeerakathil T, Wolf PA, Beiser A, et al. Stroke risk profile predicts white matter hyperintensity volume: the Framingham Study. Stroke 2004;35:1857-61

26. Rost NS, Rahman R, Sonni S, et al. Determinants of white matter hyperintensity volume in patients with acute ischemic stroke. J Stroke Cerebrovasc Dis 2010;19:230-35

27. Auriel E, Bornstein NM, Berenyi E, et al. Clinical, radiological and pathological correlates of leukoaraiosis. Acta Neurol Scand 2011;123:41-47

28. Leifer D, Buonanno FS, Richardson EP, Jr. Clinicopathologic correlations of cranial magnetic resonance imaging of periventricular white matter. Neurology 1990;40:911-18

29. van Swieten JC, van den Hout JH, van Ketel BA, et al. Periventricular lesions in the white matter on magnetic resonance imaging in the elderly: a morphometric correlation with arteriolosclerosis and dilated perivascular spaces. Brain 1991;114:761-74

30. Fazekas F, Kleinert R, Offenbacher H, et al. Pathologic correlates of incidental MRI white matter signal hyperintensities. Neurology 1993;43:1683-89

31. Moody DM, Santamore WP, Bell MA. Does tortuosity in cerebral arterioles impair down-autoregulation in hypertensives and elderly normotensives? A hypothesis and computer model. Clin Neurosurg 1991;37:372-87

32. Spangler KM, Challa VR, Moody DM, et al. Arteriolar tortuosity of the white matter in aging and hypertension: a microradiographic study. J Neuropathol Exp Neurol 1994;53:22-26

33. Pantoni L, Garcia JH. Pathogenesis of leukoaraiosis: a review. Stroke 1997;28:652-59

34. De Reuck J. The human periventricular arterial blood supply and the anatomy of cerebral infarctions. Eur Neurol 1971;5:321-34

35. Bogousslavsky J, Regli F, Uske A. Leukoencephalopathy in patients with ischemic stroke. Stroke 1987;18:896-99

36. Pu Y, Liu L, Zou X, et al. Relationship between leukoaraiosis and cerebral large artery stenosis. Neurol Res 2009;31:376-80

37. Liao D, Cooper L, Cai J, et al. Presence and severity of cerebral white matter lesions and hypertension, its treatment, and its control: the ARIC StudyAtherosclerosis Risk in Communities Study. Stroke 1996;27:2262-70

38. Dufouil C, de Kersaint-Gilly A, Besancon V, et al. Longitudinal study of blood pressure and white matter hyperintensities: the EVA MRI Cohort. Neurology 2001;56:921-26

39. Ostrow PT, Miller LL. Pathology of small artery disease. Adv Neurol 1993;62:93-123

40. Feldmann E, Daneault N, Kwan E, et al. Chinese-white differences in the distribution of occlusive cerebrovascular disease. Neurology 1990;40:1541-45

41. Wityk RJ, Lehman D, Klag M, et al. Race and sex differences in the distribution of cerebral atherosclerosis. Stroke 1996;27:1974-80

42. Yasaka M, Yamaguchi T, Shichiri M. Distribution of atherosclerosis and risk factors in atherothrombotic occlusion. Stroke 1993;24:206-11

43. Wong KS, Huang YN, Gao S, et al. Intracranial stenosis in Chinese patients with acute stroke. Neurology 1998;50:812-13

44. Li H, Wong KS. Racial distribution of intracranial and extracranial atherosclerosis. J Clin Neurosci 2003;10:30-34

45. Altaf N, Daniels L, Morgan PS, et al. Cerebral white matter hyperintense lesions are associated with unstable carotid plaques. Eur J Vasc Endovasc Surg 2006;31:8-13

46. Stary HC, Chandler AB, Dinsmore RE, et al. A definition of advanced types of atherosclerotic lesions and a histological classification of atherosclerosis: a report from the Committee on Vascular Lesions of the Council on Arteriosclerosis, American Heart Association. Circulation 1995;92:1355-74

47. Bash S, Villablanca JP, Jahan R, et al. Intracranial vascular stenosis and occlusive disease: evaluation with CT angiography, MR angiography, and digital subtraction angiography. AJNR Am J Neuroradiol 2005;26:1012-21 